\title{
Transmission Expansion Planning Considering Wind Energy Conversion Systems Using PSO
}

\author{
K. Indira, B. Srinivasa Rao
}

\begin{abstract}
In power system studies the most important issue is Transmission Expansion Planning (TEP). The intend of TEP problem is to choose the placement as well as number of additional transmission lines, which are to be added to the existing system to suit growing demand in planning horizon. In this paper a new methodology for TEP is proposed, the presented Transmission planning is linked with generation cost, active power loss minimization by considering wind uncertainties. Firstly, the uncertainties involved in wind generation can be determined by using weigbull probability functions. Monte Carlo simulation study is able to be used to find the probability distribution functions of wind generation. Then, in TEP formulation the WTG uncertainties are considered. Particle swarm optimization (PSO) technique is used for solving the proposed single objective optimization problem. Simulation studies conducted on an IEEE 30 bus test system to certify effectiveness of the TEP problem with considering wind uncertainties.
\end{abstract}

Keywords : Transmission expansion planning (TEP); probability density function; Monte-Carlo simulation; Particle swarm optimization (PSO); Wind energy systems (WES).

\section{INTRODUCTION}

The main intend of Optimal Power flow (OPF) problem is to reduce the objective functions like generation cost, L-index and transmission line loss by tuning the independent variables (control) of power system. Belgin Emre, Rengin Idil [1] discussed about optimal power flow problem by using Newton Raphson based PSO. Nowadays conventional power plants are one of the causes for environmental pollution. In order to reduce the environmental pollution and $\mathrm{CO} 2$ levels, depletion of fossil fuels, renewable energy sources are the best alternative to conventional power units. Wind energy plays a major role in case of renewable energy sources. By incorporating WECS the generation cost function is not simple while in the case of cost function of normal conventional generators. The optimal power flow problem including wind energy conversion systems are proposed in [2]. The uncertainties involved in wind generation can be determined by using weigbull probability functions. The under and over estimated wind energy is associated with reserve and penalty costs respectively and these costs are also included in the Optimal power problem formulation in the case of WECS. In this paper, TEP is proposed considering OPF with WECS, TEP problem is extension of OPF problem.

Revised Manuscript Received on September 22, 2019.

* Correspondence Author

K. Indira, PG scholar, Department of Electrical and Electronics Engineering, V R Siddhartha Engineering College, Vijayawada, A.P, India

Dr. B. Srinivasa Rao, Professor , Department of Electrical and Electronics Engineering, V R Siddhartha Engineering College, Vijayawada, A.P, India
The intend of TEP is to provide the growing load demand in the future by expanding or constructing new transmission lines. In regulated environment TEP problem aims to reduce the line investment cost only which is different from deregulated environment.

Mathematical and meta-heuristic approaches are two different approaches which are used to study the TEP issue. The mathematical methods include linear and non linear programming [3] and Bender's decomposition [4]. Mathematical methods are dealing with problems like slow convergence and dimensionality issues. In order to avoid these complexities various artificial intelligence based methods have been successfully utilized to compute OPF problem. Likewise, some heuristic methods, for example Harmony Search [5], PSO [6], Genetic algorithms [7], ant colony [8] and differential evolution [9] are effectively utilized for taking care of the OPF along with TEP issue. In this paper, to work out OPF problem with consideration of WECS the PSO method is used. To test the effectiveness of the proposed method with different cases by considering TEP IEEE-30 bus test system is used.

\section{PROBLEM FORMULATION OF OPF}

The OPF main aim is to optimize the specific objective function such as generation cost, active power loss, emission and L-index by optimal tuning of the independent variables of power system [10], while fulfilling a all the equality as well as inequality constraints. OPF problem formulation is mathematically demoted as

$\operatorname{Min} f(x, u)$

Subjected to: $\quad y(X, U)=0$

$$
z(X, U) \leq 0
$$

where $y$ is equality constraint, $\mathrm{z}$ is the inequality constraint. Equality constraints: The total generation must be equal towards the summation of total power demand and transmission losses of power system.

$$
\sum_{\mathrm{i}=1}^{\mathrm{n}} P_{g \mathrm{i}}=P_{D}+P_{\mathrm{L}}
$$

where $P_{\text {oi }}-i^{\text {th }}$ generating units power output, the total demand of the load is $P_{d} ; P_{L}$ - transmission line loss. Inequality constraints: Inequality constraints include limits of system independent and dependent variables.

\subsection{Control variables- (independent variables)}

The generator real powers, and switchable VAR sources of reactive power, generator bus voltages, tap settings of transformer. 
$U^{T}=\left[P_{G 2} \ldots . P_{G N G}, Q_{C 1} \ldots, Q_{C N G}, V_{G 1} \ldots, V_{G N G}, T_{1} \ldots, T_{N T}\right]$

(5)

where $\mathrm{u}$ is control vector which consist of all independent variables

$P_{G i}^{\min } \leq P_{G i} \leq P_{G i}^{\max }{ }_{,} i=1_{x \ldots \ldots} N G$

$P_{G i}$ is the $i^{\text {th }}$ generator real power output except at the slack bus varying from minimum to maximum values.

$Q_{\mathrm{ci}}^{\min } \leq Q_{\mathrm{ci}} \leq Q_{\mathrm{ci}}^{\max }{ }_{s} i=1_{n \ldots \ldots} \ldots$ sh

where $Q_{c i}$ is the shunt VAR compensations, where $N$ sh is the number of shunt VAR compensators.

$V_{G i}^{\min } \leq V_{G i} \leq V_{G i}^{\max }{ }_{0} i=1_{w \ldots \ldots} N G$

where $V_{G i}$ is the $i^{\text {th }}$ generator voltage varying within the limits; $N G$ - is the number of generators.

$T_{i}^{\min } \leq T_{i} \leq T_{i}^{\max }{ }_{s} i=1_{w \ldots \ldots} N T$

where $T_{1}$ is the tap setting of transformer varying within the limits; $N T$ - is the number of transformers.

\subsection{Dependent variables (State variables)}

State variables of power system are also slack bus real power, voltages at load buses, reactive powers of generator and transmission line flows.

$X^{T}=\left[P_{G 1}, Q_{G 1} \ldots, Q_{G N G}, V_{L 1} \ldots V_{L d}, S_{L 1} \ldots S_{L N L}\right]$

where dependent variables vector is represented by $\mathrm{X}$ $P_{G 1}^{\min }<P_{G 1}<P_{G 1}^{\max }$

$P_{G 1}^{\max }{ }_{n} P_{G 1}^{\min }$, are the maximum and minimum limits of slack bus real powers.

$Q_{G i}^{\min } \leq Q_{G i} \leq Q_{G i}^{\max }{ }_{0} i=1_{\ldots \ldots} \ldots N G$

$Q_{G i}$ is the reactive power of $i^{\text {th }}$ generating unit

(13)

where $V_{L i}$ is the $i^{\text {th }}$ bus voltage varying from minimum to maximum limits, $N_{\mathbb{R}}$ is the number of load buses.

$S_{L i} \leq S_{L i}^{\max }{ }_{0} i=1_{s \ldots} N L$

$S_{L i}^{\max }$ - the $i^{\text {th }}$ transmission line flow limit, $N L$ - number of transmission lines.

Security constraints: Generator reactive power limits, load bus voltage limits and transmission line flow limits are considered as security constraints. In this paper to mitigate the security constraints violations the penalty function method [11] is used i.e. the violated values of security constraints are added to objective functions.
$V_{L i}^{\min } \leq V_{L i} \leq V_{L i}^{\max }{ }_{,} i=1_{p \ldots \ldots} N_{i d}$

\section{WIND ENERGY SYSTEMS (WES)}

The optimum power flow problem incorporating wind uncertainties is discussed in this section. By incorporating non-conventional sources like wind energy, cost function of system is not simple we have to include wind generation cost function into the traditional OPF problem formulation. The mathematical formulation of cost functions including wind energy conversion systems are given below. Due to of the uncertainties of wind speed at any given time, cost function of wind generation consists of two components along with direct cost function. They are penalty cost function and reserve cost function. The over estimated cost of available wind power generation related to reserve cost. The under estimation of available wind power generation is related to penalty cost. The reserve cost function can be mathematically formulated as

$C_{\text {regi }}=k_{v_{i} i} \int_{0}^{W_{g i}}\left(W_{g i}-W_{a v b}\right) f W(W) d W$

where $k_{\gamma_{i} i}$ is the penalty coefficient of $i^{\text {th }}$ wind generating unit.

The penalty cost function can be mathematically formulated as

$C_{p e n, i}=k_{p, i} \int_{w w_{g i}}^{w_{r i} i}\left(W_{a v b}-W_{g i}\right) f W(W) d W$

Where $k_{p i i}$ is the penalty coefficient of $i^{\text {th }}$ wind generating unit; $f W(W)$ is the wind power PDF; $w_{g i}$ is the power from $i^{\text {th }}$ wind power generating unit; $W_{\mathrm{i}_{\mu}} w_{\mathrm{vb}}$ is the wind power availability of $i^{\text {th }}$ wind generator this is a random value varying from 0 to $w_{\gamma}$, and probability of $w_{y}$ is varying with probability Density function. The PDF of wind power output can be determined by using Monte Carlo simulation method, based on the Weibull distribution of wind where $w_{y}$ is the rated wind power; $C_{i}$ is the cost function for $i^{\text {th }}$ thermal generating unit; $C_{\text {खgi }}$-the cost function for $i^{\text {th }}$ wind unit; $C_{\text {peni }}$ is the penalty cost function; $C_{\text {regi }}$ is the reserve cost function related with penalty due to over estimation of available wind power. For tracking the wind uncertainties a probabilistic wind model is a very suitable model. At a certain location the probability density function (PDF) of wind speed is generally obtained by Weibull distribution. In Weibull function the shape and scale parameters are present which are obtained by using the mean wind speed and standard deviation (SD) of the wind speed [12]. Wind speed consists of three parameters, they are cut in speeds $\left(V_{c i}\right)$, cut out speeds $\left(V_{c o}\right)$ and rated speeds $\left(V_{\text {rate }}\right)$ are involved in estimation of power output characteristics.

\section{TRANSMISSION PLANNING}

TEP can be done by building new transmission lines with less cost, which change active and reactive power flow through the lines which already exist in power system network. Two network topologies are present to solve TEP problem.

-Existing network (which consists of existing transmission lines) 
-Modified network (consist of new transmission lines) to satisfy the growing load demand in future.

In this paper for solving TEP problem the load on the IEEE-30 bus system is increased by $80 \%$ and then the line flow violations are observed and corresponding lines are selected to mitigate the line flow violations.TEP problem is a non-linear mixed integer program. Garver [13] has proposed the use of linear programming technique for solving transmission expansion planning problem. Different models available for representing the transmission networks. There are four major types of network models have been used for representing the power system network in TEP studies [14]. They are DC power flow [15], transportation model, hybrid model and disjunctive model. The use of above model have the drawback that the transmission planning problem is different from reactive power planning problem so in this paper an AC TEP is considered where network reactive powers also included. $\mathrm{Mj}$ rider and $\mathrm{R}$. Romero has been proposed [16] an accurate AC network modelling.

\subsection{Mathematical modeling of AC TEP:}

AC TEP can be mathematically formulated as by eqs (17) to (24).

$$
\min _{\text {s.t }} v_{t}=c^{t} n
$$

$$
P(v, n)-P_{G}+P_{d}=0
$$

$$
\begin{aligned}
& Q(v, n)-Q_{a}+Q_{d}=0 \\
& \left(N^{e x}+N^{b}\right) S^{\text {from }} \leq\left(N^{e x}+N^{b}\right) S^{0}
\end{aligned}
$$

$$
\left(N^{a x}+N^{b}\right) S^{\text {to }} \leq\left(N^{a x}+N^{b}\right) S^{0}
$$

while $\mathrm{c}$ is the transmission lines cost vector, $\mathrm{n}$ is the added new lines. $\mathrm{N}^{\mathrm{ex}}$ and $\mathrm{N}^{\mathrm{b}}$ denotes diagonal matrices consist of transmission lines in case of expanded and base case respectively. $v_{t}$ - line investment cost because of the building of new lines to the power system network. Where $P_{G}, Q_{G}$ and $P_{d}, Q_{d}$ are the active and reactive power vectors of generation and load. $\mathrm{V}$ represents the voltage vector. $S^{\text {from }}, S^{\text {to }}$, and $S^{0}$ - vectors of complex power flows and their maximum power flow limits.

\subsection{Objective functions}

(i) Minimization of total generation cost of a system is expressed as

Min $f_{1}=f_{1 c}+f_{1 w}$
where $f_{1 e}=\sum_{i=1}^{N}\left(a_{i} P_{g i}^{2}+b_{i} P_{g i}+c_{i}\right)$

$$
f_{1 w}=\sum_{i}^{\mathrm{Nw}}\left(C_{\text {diri }}+C_{\text {resi }}+C_{\text {veni }}\right)
$$

where $f_{1 c}$ the cost function for conventional generators is, $f_{1 w}$ is the cost function for wind generators. $N w$ is the number of wind units. $a_{\mathrm{i}}, b_{\mathrm{i}}, c_{\mathrm{i}}$ are the generator cost coefficients for $i^{\text {th }}$ generating unit, $\mathrm{n}$ is the number of thermal units, and $P_{\text {oi }}-$ power output of the $i^{\text {th }}$ unit. If the WECS units in system are absent then cost function $f_{1 e}$ only exists.

(ii) Minimization of active power loss

$$
f_{2}=\sum_{k=1}^{N L} G_{k}\left[v_{g}^{2}+v_{T}^{2}-2 v_{g} v_{Y} \cos \left(\theta_{g}-\theta_{T}\right)\right]
$$

where $G_{k}$ is the conductance of transmission line, $\theta_{g}$ and $\theta_{r}$ are the angles of buses, $v_{g}, v_{r}$ are the voltages at bus $\mathrm{s}$ and $\mathrm{r}$.

\section{METHODOLOGY}

PSO is the heuristic optimization technique which was developed in 1995 by Eberhort and Kennedy. It is a well familiar method known to resolve comprehensive non linear problems effectively and it is one of the classifications of the Evolutionary computations [17], used to resolve the problems of optimization. For the every iteration, the velocity vector for each particle is in synch according to the chronological best position of that particle and the region particle best position. A solution which is optimal or near optimal is obtained to the natural moment of the particles and it can generate good quality solutions with a reduced amount of computational time and more constant convergence characteristics, where most analytical methods fail to converge. The velocities and positions are keeping up to date for all the particles using the following equations.

$$
\begin{aligned}
& V_{m}{ }^{k+1}=w_{f} * V_{m}{ }^{k}+c_{p 1} * r_{p 1} *\left(p_{b}{ }^{k}-X_{m}{ }^{k}\right)+c_{g 2} * r_{g 2} * \\
& \left(G_{b}{ }^{k}\right) \\
& \left.-X_{m}{ }^{k}\right)
\end{aligned}
$$

$X_{m}^{k+1}=X_{m}^{k}+V_{m}^{k+1}$

Where $\mathrm{k}$ is the iteration value, $V_{\mathrm{m}}{ }^{k}$ velocity of a particle at $k^{\text {th }}$ iteration, $X_{m}{ }^{k}$ is the location of particle at $k^{\text {th }}$ iteration. Where stopping criteria is exceeding maximum iterations or good fitness value is obtained. Here generation cost \& power loss of system can be solved by using PSO.

\section{SIMULATION RESULTS}

The effectiveness of proposed method is verified by MATLAB programming. To resolve the optimal power flow problem by considering WECS with TEP a standard IEEE 30 bus test system is used. This test system consists of six generating units, 41 transmission lines, four tap changing transformers, nine shunt capacitors, the minimum and maximum limits of generator and generation cost coefficients are taken from [1]. Load demand of the system is defined as 2.8340p.u. Total population size is chosen as 50 and number of particles is 25 which include Pg's, Vg's, Taps and Q shunts. The test system results are compared for before and after TEP. The minimization of generation cost, active power loss is taken as two different objectives for the following cases.

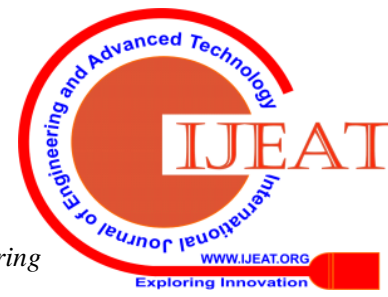




\section{Case 1: Without WECS}

Case 2: With WECS

In case of WECS the conventional generators at buses 4, 5 and 6 are replaced with wind generating units. The cut in speed, rated speed and cut out speed of given system are taken as $3 \mathrm{~m} / \mathrm{s}, 12 \mathrm{~m} / \mathrm{s}$ and $30 \mathrm{~m} / \mathrm{s}$ respectively.

\subsection{Before considering TEP}

6.1.1 Case 1- Without WECS: The convergence plots of IEEE 30 bus test system for cost minimization and loss minimization are in Fig 1.(a) and Fig 1.(b) respectively. Before optimization the generation cost and power loss are 900.59(\$/hr), 5.32MW. By performing OPF using PSO, the total generation cost and active power loss of the system is reduced to $799.5408(\$ / \mathrm{hr})$ and 3.05MW. Optimal values of the power generations corresponding to cost \& power loss minimization are given in Table 1 .

Table 1: Optimization results for generation cost minimization, active power loss without TEP

\begin{tabular}{|c|c|c|c|c|c|c|}
\hline Case & \multicolumn{3}{|c|}{ Case 1} & \multicolumn{3}{|c|}{ Case 2} \\
\hline Independent variables & Base case & $\begin{array}{l}\text { Cost } \\
\text { objective }\end{array}$ & $\begin{array}{l}\text { Loss } \\
\text { objective }\end{array}$ & Base case & Cost objective & Loss objective \\
\hline $\mathrm{pg}_{1}(\mathrm{MW})$ & 98.727 & 177.13 & 53.04 & 19.36 & 94.252 & 52.195 \\
\hline $\mathrm{pg}_{2}(\mathrm{MW})$ & 80 & 48.696 & 78.40 & 80 & 28.991 & 36.544 \\
\hline $\mathrm{pg}_{3}(\mathrm{MW})$ & 20 & 21.114 & 35.00 & 20 & 10 & 35 \\
\hline $\mathrm{pg}_{4}(\mathrm{MW})$ & 20 & 11.904 & 30.00 & 50 & 60 & 60 \\
\hline $\mathrm{pg}_{5}(\mathrm{MW})$ & 50 & 21.315 & 50.00 & 57 & 39.452 & 60 \\
\hline $\mathrm{pg}_{6}(\mathrm{MW})$ & 20 & 12 & 40.00 & 60 & 54.856 & 41.856 \\
\hline$\sum p g_{\mathrm{i}}(\mathrm{MW})$ & 288.72 & 292.16 & 286.45 & 286.3 & 292.16 & 286.45 \\
\hline $\operatorname{Cost}(\$ / h r)$ & 900.59 & 799.5408 & 964.07 & 869.5615 & 785.83 & 822.46 \\
\hline Power loss (MW) & 5.32 & 8.76 & 3.05 & 2.96 & 4.1498 & 2.194 \\
\hline
\end{tabular}

6.1.2 Case 2- With WECS: The Convergence plots of IEEE-30 bus test system for cost minimization and loss minimization by including WECS are shown in Fig 1.(c) and Fig 1.(d) respectively. Before optimization the total generation cost and power loss are $869.5615(\$ / \mathrm{hr})$ and $2.96 \mathrm{MW}$ by performing OPF using PSO, the total generation cost and active power losses of test system is reduced to $785.83(\$ / \mathrm{hr})$ and $2.194 \mathrm{MW}$ respectively. Optimal values of the power generations corresponding to cost and power loss minimization are given in Table 1 .

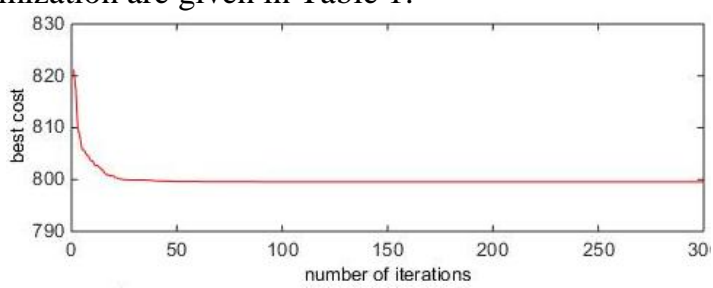

(a) Cost minimization without TEP (Case 1)

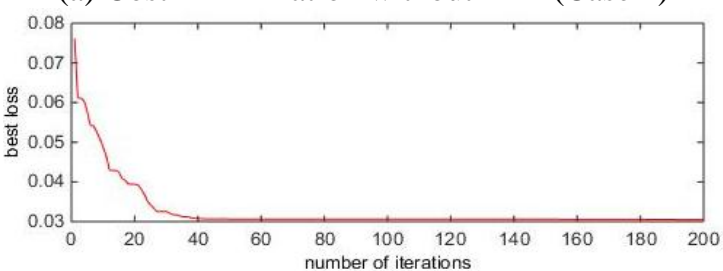

(b) Loss minimization without TEP (Case 1)

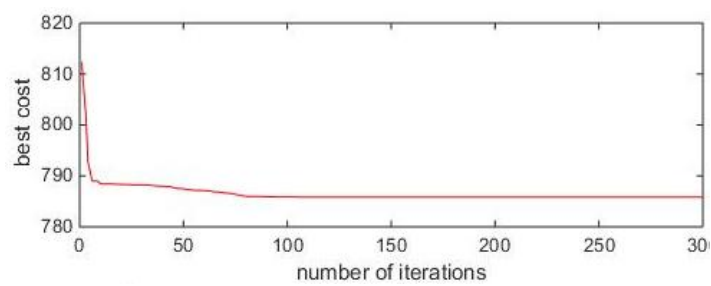

(c) Minimization of cost without TEP (Case 2)

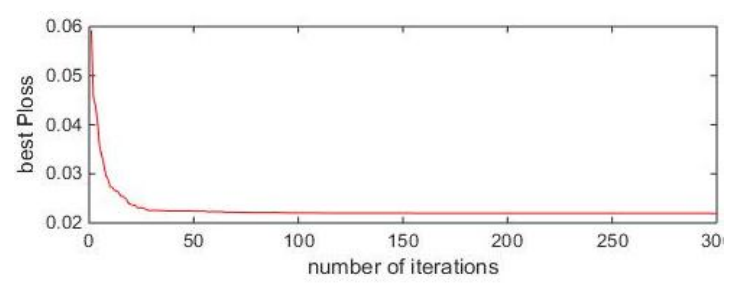

(d) Loss minimization without TEP (Case 2)

Fig 1. Convergence characteristics for cost minimization and loss minimization without TEP

\subsection{After considering TEP}

The load at various buses of standard IEEE-30 bus test system is increased by $20 \%, 40 \%, 60 \%$ and $80 \%$ of the base case in order to identify line flow violations. The detailed results of each load demand are shown in Table 2 . There is no line flow violation for $20 \%$ load enhancement; line 1-2 is violated for $40 \%$ load enhancement. For $60 \%$ load increment the lines 1-2, 2-13, 13-3, and for $80 \%$ load increment the lines $1-2,2-13,11-13,13-3,12-15$ are violated their maximum power limits. It can be observed that with $80 \%$ load enhancement there are 5 lines violating their limits. In order to overcome this, TEP problem is solved by considering 9 candidate lines for the existing IEEE 30 bus system as shown in Table 3. 
Table 2: Effect of load change in IEEE - 30 bus test system

\begin{tabular}{llllll}
\hline Independent variables & Base case & $20 \%$ load added & $40 \%$ load added & $60 \%$ load added & $80 \%$ load added \\
\hline $\mathrm{pg}_{1}(\mathrm{MW})$ & 98.73 & 160.02 & 223.25 & 292.76 & 356.17 \\
$\mathrm{pg}_{2}(\mathrm{MW})$ & 80 & 8 & 80 & 80 & 80 \\
$\mathrm{pg}_{3}(\mathrm{MW})$ & 20 & 2 & 20 & 20 & 20 \\
$\mathrm{pg}_{4}(\mathrm{MW})$ & 20 & 2 & 20 & 20 & 20 \\
$\mathrm{pg}_{5}(\mathrm{MW})$ & 50 & 5 & 50 & 50 & 50 \\
$\mathrm{pg}_{6}(\mathrm{MW})$ & 20 & 2 & 20 & 20 & 20 \\
$\Sigma \mathrm{pg}_{\mathrm{i}}(\mathrm{MW})$ & 288.73 & 350.02 & 413.25 & 478.57 & 546.17 \\
Load $(\mathrm{MW})$ & 283.4 & 340.08 & 396.76 & 453.44 & 510.12 \\
Overloaded lines & - & - & 1 & 3 & 5 \\
\hline
\end{tabular}

Table 3: Added transmission line details and cost for transmission expansion planning

\begin{tabular}{l|cccccc}
\hline Corridor & $2-13$ & $11-13$ & $12-15$ & $10-21$ & $13-3$ & $1-2$ \\
Number of lines added (9) & 2 & 2 & 1 & 1 & 1 & 2 \\
Total cost (\$) & \multicolumn{7}{|c|}{24206} \\
\hline
\end{tabular}

The total investment cost for given planning problem is $24206 \$$. Now by considering this modified IEEE 30 bus test system with inclusion of new lines the following case studies conducted.

\subsubsection{Case 1- Without WES:}

The Convergence characteristics for cost minimization and loss minimization without WECS by considering TEP problem are in Fig 2. (a) \& Fig 2. (b) respectively. By

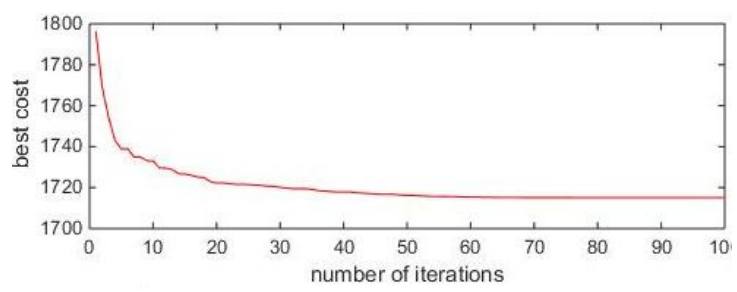

(a). Cost minimization with TEP (Case 1)

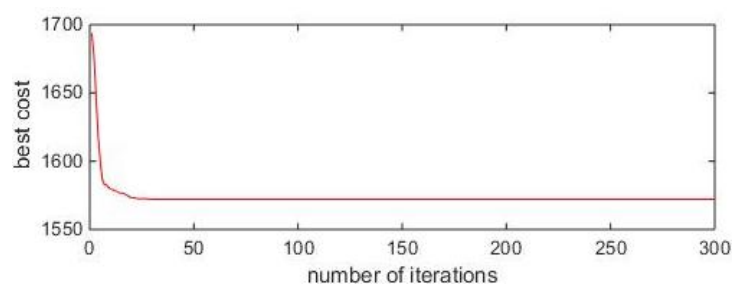

(c). Cost minimization with TEP (Case 2) considering TEP before optimization the fuel cost is $1794(\$ / \mathrm{hr})$, active power loss is $22.89 \mathrm{MW}$. By performing OPF with TEP problem using PSO, total generation cost and active power loss of test system is decreased to $1714(\$ / \mathrm{hr})$ and 7.7MW respectively. The minimization of cost and power loss optimal values are shown in below Table 4 .

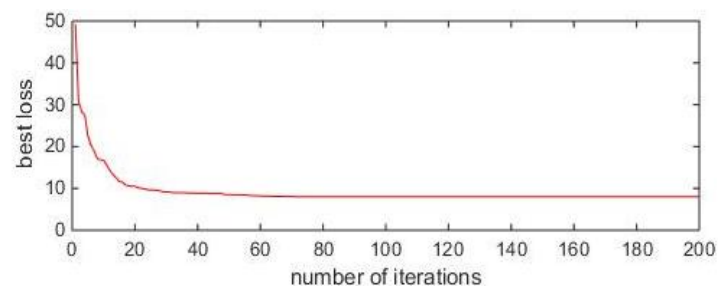

(b). Loss minimization with TEP (Case 1)

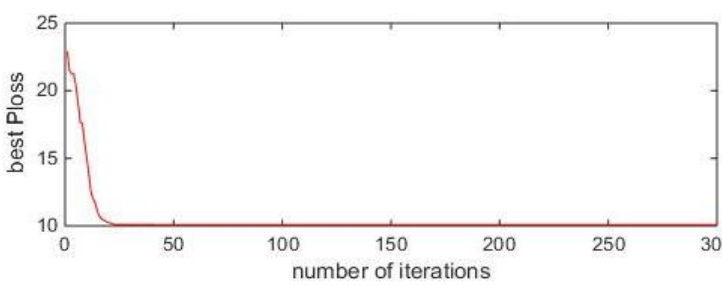

(d). Loss minimization with TEP (Case 2)

Fig 2. Convergence plots for TEP

\subsubsection{Case 2 - With WES:}

The convergence characteristics for generation cost minimization \& loss minimization with WECS by taking into consideration TEP problem are shown fig Fig.2. (c) \& 2. (d) respectively. Before optimization the cost and power loss are 1787.77(\$/hr), 21.79MW. By performing OPF considering
TEP with WES using PSO, the generation cost \& active power loss of test system is minimized to $1561(\$ / \mathrm{hr})$ and $10.1 \mathrm{MW}$ respectively. The optimum values of power generations corresponding to cost and power loss are given in Table 4.

Table 4: Optimization results for TEP without and with WES

\begin{tabular}{l|lll|llc}
\hline \multirow{2}{*}{ Independent variables } & \multicolumn{3}{|c|}{ Case 1 } & \multicolumn{3}{c}{ Case 2 } \\
\cline { 2 - 7 } & Base case & $\begin{array}{l}\text { Cost } \\
\text { objective }\end{array}$ & $\begin{array}{l}\text { Loss } \\
\text { objective }\end{array}$ & Base case & Cost objective & Loss objective \\
\hline $\operatorname{pg}_{1}(\mathrm{MW})$ & 343.11 & 292.76 & 101.98 & 341 & 237.64 & 133.18 \\
$\operatorname{pg}_{2}(\mathrm{MW})$ & 8 & 72.985 & 144 & 80 & 60.475 & 144 \\
\hline
\end{tabular}




\begin{tabular}{l|lll|lll}
\hline $\mathrm{pg}_{3}(\mathrm{MW})$ & 2 & 72.907 & 63 & 20 & 44.249 & 63 \\
$\mathrm{pg}_{4}(\mathrm{MW})$ & 2 & 29.539 & 54 & 20 & 60 & 60 \\
$\mathrm{pg}_{5}(\mathrm{MW})$ & 5 & 30.083 & 90 & 50 & 60 & 60 \\
$\mathrm{pg}_{6}(\mathrm{MW})$ & 2 & 28.942 & 64.77 & 20 & 60 & 60 \\
$\Sigma \mathrm{pg}_{\mathrm{i}}(\mathrm{MW})$ & 533.01 & 527.2 & 517.82 & 531.9 & 522.3 & 520.2 \\
Cost $(\$ / \mathrm{hr})$ & 1794 & $\mathbf{1 7 1 4}$ & 2225 & 1787.77 & $\mathbf{1 5 6 1}$ & 1729.3 \\
Power loss $(\mathrm{MW})$ & 22.89 & 17.1 & $\mathbf{7 . 7}$ & 21.79 & 12.25 & $\mathbf{1 0 . 1}$ \\
\hline
\end{tabular}

\section{CONCLUSION}

In this paper an attempt has been made to solve TEP problem using PSO with consideration of WES. Initially the OPF is solved using PSO generation cost minimization \& power loss reduction. Later the effect of WECS is taken with consideration for implementing OPF problem. Here the generator cost function is not simple by incorporating WECS. Here penalty cost and reserve cost, which are associated with over and under evaluation of wind energy are included in the generator cost function. For IEEE 30 bus system the TEP analysis carried at by increasing $80 \%$ of the load on the system and total line cost is also determined for newly added lines. Then the OPF problem is implemented for TEP problem with and without WECS. The proposed methodology can be extended for implementing multi objective optimization problem using different methods with TEP by considering WECS.

\section{REFERENCES}

1. Belgin emre turkay, Rengin idil cabadag, "Optimal Power Flow Solution Using Particle Swarm Optimization Algorithm”, EuroCon 2013.

2. L. B. Shi, C. Wang, L. Z. Yao, L. M. Wang, Y. X. Ni, B. Masoud, "Optimal Power Flow with Consideration of Wind Generation "Cost, International Conference on Power System Technology 2010.

3. Al-Hamouz ZM, Al-Faraj AS. "Transmission expansion planning based on a nonlinear programming algorithm". Applied Energy 2003;76:169-77.

4. T. Akbari, A. Rahimikian, A. Kazemi.,"A multi-stage stochastic transmission expansion planning method". Energy Conversion and Management 2011;52:2844-53.

5. A. Verma, BK.. Panigrahi, PR. Bijwe,"Harmony search algorithm for transmission network expansion planning". IET Generation, Transmission and Distribution 2010;4:663-73.

6. H. Shayeghi, M. Mahdavi, A. Bagheri, "Discrete PSO algorithm based optimization of transmission lines loading in TNEP problem”. Energy Conversion and Management 2010;51:112-21

7. S. Jalilzadeh, A. Kazemi, H. Shayeghi and M. Madavi, "Technical and economic evaluation of voltage level in transmission network expansion planning using GA". Energy Conversion and Management 2008;19:1119-25.. Energy Conversion and Management 2011;52:382-90.

8. AML. Da Silva, LS. Rezende, Da Fonseca Manso LA, De Resende LC. Reliability worth applied to transmission expansion planning based on ant colony system. Electrical Power and Energy Systems 2010;32:1077-84.

9. PS. Georgilakis, "Market-based transmission expansion planning by improved differential evolution". Electrical Power and Energy Systems 2010;32:450-6.

10. M.A. Abido, "Optimal power flow using tabu search algorithm", Electric Power Components and Systems, Taylor \& Francis Group pp. 30:469-483, 2002.

11. Xian Liu," Minimum Emission Dispatch Constrained by Stochastic Wind Power Availability and Cost". IEEE Transactions On Power systems 2010:25:3.

12. B. Srinivasa Rao, A. Bala Naga Lingaiah, "Solving Multi Objective ORPD Problem Using AIS Based Clonal Selection Algorithm with UPFC". Journal of electrical system 2017, 13-1: 27-42.
13. L. L. Garver, "Transmission Network Estimation Using Linear Programming," IEEE Transaction on Power Sys- tems, Vol. 89, No. 8, 1970, pp. 2025-2034.

14. Karunya, 1., P.Harini, ., S.Iswarya, . \& A.Jerlin, . (2019) emergency alert security system for humans. International journal of communication and computer technologies, 7 (supplement 1), 6-10. Doi:10.31838/ijccts/07.sp01.02

15. M. J. Rider, A. V. Garcia and R. Romero, "Power System Transmission Network Expansion Planning Using AC Model," IEE Proceedings Generation, Transmission and Distribution, Vol. 1, No. 5, 2007, pp. 731-742. doi:10.1049/iet-gtd:20060465.

16. Yamille delValle, Ganesh Kumar Venayaga moorthy, Salman Mohagheghi," Particle Swarm Optimization: Basic Concepts, Variants and Applications in Power Systems". IEEE Transactions On Evolutionary Computation 2008:12:2 